\title{
A Novel Fast Decision-making Method Based on Integrated Intelligent Technologies for Wind Disaster Management of Energy Manufacture
}

\author{
Baishang Zhang ${ }^{1}$, Jincheng Hong ${ }^{2}$, Xinyuan $\mathrm{Li}^{3}$, Jianxin Cheng, ${ }^{2, *}$ \\ ${ }^{1}$ Development Research Center, State Administration for Market Regulation of China, Beijing, PR China \\ ${ }^{2}$ School of Public Administration \& Emergency Management, Jinan University, Guangzhou, PR China \\ ${ }^{3}$ School of College of Basic Medicine, Jinan University, Guangzhou, PR China \\ *corresponding author.
}

\begin{abstract}
Energy manufacture is very important to all of industries. Typhoons hit the power grid in China's southeast coastal areas frequently for the past few years, seriously affecting the industries' operation. Therefore, making-decision of wind damage management for nation's electricity grid in real time is an urgent subject to be studied. The traditional decision making method is easy to be implemented, but is not proper for dealing with nonlinear problems in complex systems. The purpose of this article is to design a fast decision making framework for accomplishing fast decision making by making combination Case-Based Reasoning (CBR) with Rule-Based Reasoning (RBR), Genetic Algorithm (GA), which is called fast decision making method based on integrated intelligent technologies (FDMMBIIT). Compared with traditional methods, FDMMBIIT completes case adaptation with BPNN after extending case base. To make the decision-making more accurate, this article updated the multi-object genetic algorithm (MOGA) with adaptive genetic operators and a selection method by using the fitness function. Likewise, $B P N N$ is improved with adaptive simulated annealing algorithm (ASAA), which is named as BPNNASAA. More important, this paper expands the frame theory by integrating it to the D/S evidence theory, exploring a novel solution to representing cases with incomplete information. The case of Guangdong demonstrates FDMMBIIT achieves better decision-making performance for storm disaster emergency management.
\end{abstract}

Keywords: Disaster management, Energy manufacture, industry, case-based reasoning, multi-object genetic algorithms, back-propagation neural network

\section{Introduction}

In recent years, extreme weather and geological disasters occur frequently in China, threatening the security of the power grid greatly. It is undeniable that various natural disasters come one after another more frequently than ever before, which begins with the happening of freezing disaster in southern China in early 2008. Especially, Typhoon disasters hit the power grid in China more frequently, among which HAIKUI in August 2012, Fitow in October 2013, Rainbow in October 2015, do large damage on economic development and residents' life in China. The typhoon disasters often result in lines disconnection, tower damage and blackouts, making power grid wind disaster emergency a critical subject for decision makers for implementing rescue and repair work.

Because the power grid emergency is characterized by complex, urgency, less cases and incomplete information, traditional methods of decision-making could not work normally for wind disaster emergency of power grid. CBR is an intelligent decision-making method according to the current state, which has strong learning ability for providing decision support for nonlinear problems. However, implementing case adaptation in the CBR is a challenging task because the task requires calculating the type and number of emergency resources and emergency personnel. In general, calculation in case adaptation can be achieved by adjusting models, GA and k-NN [1-3]. Specifically, KNN method depends on domain knowledge, which is easy to be implemented. However, the accuracy of adaptation results of this method is often low; GA is a global optimization method in complex systems, which has advantages in case adaptation. However, it is usually a long time to achieve the object of optimization

ISSN: 0010-8189

(C) CONVERTER 2021

Www.converter-magazine.info 
with GA, missing the requirement of real-time decision making; BPNN is also being studied as a kind of adjustment method, which aims to obtain the correlations between scenario features and decision features by minimizing the network error iteratively. In consideration of the advantages and disadvantages of above methods, this paper proposes FDMMBIIT to accomplish quick decisions in power grid emergency management. In FDMMBIIT, CBR, GA module and BPNN module are integrated with together to constitute an intact emergency decision-making system, in which CBR is the main part of the system and in charge of case representation, case retrieving and case base maintenance. For GA module, it is designed to accomplish the task of extending case base and BPNN module is studied to make fast decision-making based on the training results from expanded cases. The innovations of the article are:

This paper proposes a novel quick decision making methodology for wind damage emergency management by integrating CBR, GRAMOGA and BPNNASAA together to form a whole decision making unity, which has more advantages than traditional decision making methods in dealing with complex non-line system problem, fast decision making and incomplete information.

The paper proposes a case adaptation method by building 30 BPNNASAA models and forming a parallel structure of BPNNASAA, which enriches the method of case adaptation in CBR.

The article designs a novel case base extending method by using MOGA to produce both qualitative case base and quantitative case base, what has specific steps and validation verification method.

(4) For making the method more accurate, the paper has done some improvements in BPNNASAA and simple GA (SGA). Specifically, BPNNSAA is modified with ASAA what can obtain the adaptive learning rate, expanding the theory of BPNN. Likely SGA is improved with adaptive genetic operators and population selection method based fitness function ranking, indicating a new method of improving the effectiveness of GA.

The paper follows the following structure. Section 2 summarizes the done research work. Section 3 elaborates the theory and method of FDMMBIIT for power grid wind damage management. Section 4 carries out the simulation experiment and evaluates the accuracy of proposed method. Section 5 makes comments on the method and puts forward the future research idea. Finally, Section 6 makes conclusion for the paper.

\section{Literature Review}

\subsection{The Case Adaptation of CBR}

CBR is an intelligent reasoning method proposed by Schank, which makes intelligent decision based on historical cases. The intelligent method has four processes including case representation, case retrieval, case adaptation and case retention [4-5]. Due to the fact that case adaptation is a hard task there is less research on case adaptation although much research have been conducted on case representation, case retrieval and feature-weights learning[6-8].

From some literature on case adaptation, we can find that the form of case adaptation can be divided into three types based on the different forms of problems, including case adaptation for pictures, case adaptation for words and case adaptation for data. Adaptation for pictures is is widely used in medical imaging and other fields [9]. Case adaptation for words is used to finish the adaptation task presented by words. The third type of adaptation has a wider range of applications because problems from nature and society can be represented by data. The case adaptation for data is often completed by the integration of CBR into intelligent technologies, including two kinds of adaptation methods: adaptation method based on classification and adaptation method based on computation. The method of case adaptation based on classification is usually used in diagnosis, prediction and so on, which works on the principle that CBR judges the target case falls into which category after comparing the target case to

ISSN: 0010-8189

(C) CONVERTER 2021

www.converter-magazine.info 
the history cases for making a decision [10]. Data Mining is a common method of knowledge discovery, which can obtain knowledge from things happened in the past for classification [11]. Li Hui and Sun Jie [12] distinguish that which category the target case falls into by using data mining based on Electre in the prediction of finance distress in 2009. Support Vector Machine (SVM) is a kind of new learning statistics theory for classification and regression, which works on the principle that the minimum of structure risk. In consideration of the strong ability of ANN in learning and adaptability, Pinzón Cristian et al. [13] integrates SVM with ANN for classification to complete the task of detecting and stopping the attacking of SQL. Chuang Chunling [14] constructs a BPNN classification model to judge whether a patient is suffered from liver disease or not, exploiting a novel method of case adaptation. In view of the ability of GA in classification, Lam H.Y. et al. [15] exploit GA to classify the cases on the basis that the clustering center of chromosome indicates parameter values. Although the processes and methods of these smart technologies for classification is greatly different, but they share one common what is that they judge that which category one case belongs to after data analysis and finally make a qualitative decision.

The method of case adaptation based on computation is the other important case adaptation method. This method can be classified into direct method and indirect method based on the means of solution obtaining. Specifically, indirect case adaptation obtains results after obtaining or improving models and formula, but the direct case adaptation often competes the adaptation process with intelligent technologies such as GA, ANN, k-NN and so on $[16,17]$. Due to the complexity of emergency scenario, rescue workers and emergency resources, there is neither model nor formula for the process of adaptation, resulting in the fact that direct case adaptation is fit to power grid emergency. Among the direct case adaptation methods, k-NN has the advantage of easy application because of its independence on professional knowledge, but it has low efficiency in case adaptation [18]. As for the case adaptation method based on GA, although GA can obtain the optimal value of objective function after complex process of genetic variation and optimal search, this method costs much time, causing the probability of missing the deadline for decision making in power grid emergency [19]. ANN constructs decision-making model though continuous training of the input data sets, which can meet the needs of decision makers for fast decision-making.

\subsection{Case Base Expanding}

Now there is much research on case base maintenance what aim to improve retrieval efficiency by using professional technologies [20]. However, less research is done to expand the case base scientifically and objectively what is a vital task in the field that there are not many cases.

From the less studies on case base expanding, it is concluded that the case base expanding methods could be generally divided into two categories: obtaining cases outside and generating cases inside. Generally, including contents of targeted websites into case bases is a main means of obtaining information outside, exploiting text minging, text classification and information storage [21, 22]. As for producing cases inside the system, it is known that three kinds of theories and methodologies could be used to hit the target of increasing cases. Specifically, the related theories and methodologies include Mente Carlo simulation, Rule-Based Reasoning and similarity relations theory, applying to different fields due to their application condition. The method of extending case base based on Mente Carlo simulation, which is capable of simulating the process of samples producing in given probability distribution, generates lots of random number through a great deal of simulation experiments for extending the case base[23]. The method of extending case base based on RBR judges the state, tendency, result and correlation according to the nature and internal logic of objects being studied. This method is usually applied in extending the qualitative case base rather then in dealing with quantitative case base because it often uses explicit knowledge to complete reasoning processes, which has a strong ability in explaining [24]. Finnie Gavin,Sun Zhaohao [25] propose the method of extending case base based on similarity relation according to similarity theory. There idea is that similar problems can be divided into one classification and if a problem the category can be solved, then other problems in the same category can be resolved with similar solutions even with the same solutions. Due to the fact that similarity is usually presented in a variety of forms such as words, numbers, pictures, this method can be used for both extending qualitative cases and extending quantitative cases, which has more extensive applicability[26].

ISSN: 0010-8189

(C) CONVERTER 2021

www.converter-magazine.info 
However, specific methods, clear steps and validation analysis for extending case base have not been put forward under the guidance of similarity theory.

Motivated by defects of traditional case base extending method, this paper proposes the method of extending case base based on GRAMOGA to solve the problem of inadequate cases in power grid emergency management.

\subsection{BPNN}

$\mathrm{Xu}$ M. proposed the neural network to simulate how the brain of human receives external signals and learns, during which the brain continues to adapt to the environmental rules by constantly learning and revising [27, 28]. It has been seen as a useful tool in parallel-distributed processing fields because it is characterized by parallel and distributed association memory, fault tolerance, adaptability and good learn ability [29].

BPNN is one of the most representative and widely applied model in lots of fields, during which the gradient deepest decent method is adopted to minimize the error and infer the Delta Rule [30]. The neural network process includes two parts in which the first process is called the learning process and the second one is called the recall process. During the first process, a weighted value and threshold value are obtained by using designed models; following the first process, the weighted value and threshold value are used to provide model-forecasting power

[31]. The neurons repeatedly do many turns of training with (1) and (2) to update the neuron's activation value $O_{i}$.

$$
\begin{aligned}
& \text { network }_{j}=\sum_{k=1}^{n} w_{k j} x_{k}-\theta_{j} \\
& \text { o } \left._{\text {network }}\right)=f\left(\text { network }_{j}, \text { network }_{j-1}, x_{k}\right)
\end{aligned}
$$

Despite the remarkable advantages of BPNN, two drawbacks are slow convergence and local optimization, which affect its performance to large extent. Consequently, researchers have been trying to improve the BPNN model by applying three types of approaches. The first approach aims to achieve the target of global optimization by fixing the architecture of BPNN, including tabu search method, GA and so on. The second method is determination of excellent parameter settings by combining other search methods with BPNN, learning circle fixing and suitable learning rate searching. Another improvement channel is network pruning what assumes that there are too many connections in BPNN, which can produce a new smaller BPNN after pruning some connections. The advantages of fast convergence and high accuracy can be gotten during the renewed training after combining BPNN with SA and sensitivity measurement.

It is found that heuristic algorithms have been treated as a popular method for optimizing BPNN, among which SA is usually used to determine proper learning rate because of its better ability to search for the global optimal parameters [32]. However, some improvement could also be made by adjusting the learning rate according to prediction error in real time because prediction error suggests where the learning rates should be increased or decreased. Motivated by that consideration, this paper proposes BPNNASAA for improving the performance of BPNN.

\section{Theory Models and Methodology}

3.1 Theory models

3.1.1 System Workflow

ISSN: 0010-8189

(C) CONVERTER 2021 
The process of this system can be classified into the three critical steps (see Fig. 1):

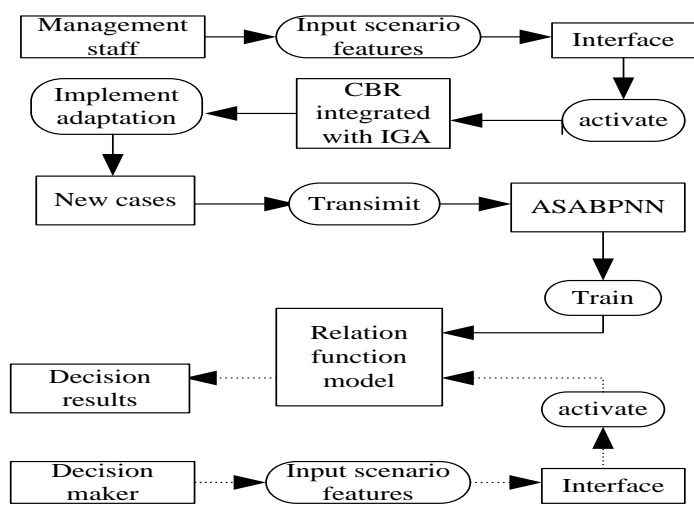

Fig.1: The chart of the system's work flow

(1) Under non-emergency environment, in consideration of improved GA's strong ability in searching for optimal solution and much time costing in the process, this system aims to extend case base by integrating MOGA with CBR based on the grey relation system theory.

(2) After expanding the case base, BPNNASAA module will be activated to determine the functional relationships between scenario features and decision features by training lots of samples and store functions in the model base.

(3) When a wind damage accident of power grid happens, the scenario features will be input into the system through the interface and the decision results will be output and displayed in the screen after invoking the stored functions.

\subsubsection{The Theory of GA for Extending Case Base}

Because one case of wind damage accident emergency of power grid include scenario features $S=\left(s_{1}, s_{2}, \cdots s_{i}\right)$ and emergency plan $P=\left(p_{i+1}, p_{i+2}, \cdots, p_{m}\right)$, one case could be represented as $\mathrm{C}=(\mathrm{S}, \mathrm{P})$. Then $\mathrm{N}$ cases make up a decision matrix $Z_{m \times n}$ what has a function relationship between scenario features and emergency plan. The function relationship can be represented as $\mathrm{P}=\mathrm{f}(\mathrm{S})$. Due to the fact that required personnel and equipment need to be computed based on the scenario features values of $\mathrm{S}$ and values of $\mathrm{P}$ are independent variables and dependent variables respectively. Sun et al [33] conducts research on extending case base based on similarity relationship in the field of marketing, holding the view that the classification fronted by a sale agent can be regarded as a partion set based on similarity relationship in the problem space. If $\mathrm{S}$ is a similar relationship in the problem space $\mathrm{Wp}$, then $[\mathrm{Pw}]=\{[\mathrm{p}] \mid \mathrm{p} \in \mathrm{Wp}\}$ is a problem set corresponding to $\mathrm{S}$ in $\mathrm{Wp}$. For two problems $\mathrm{p} 1, \mathrm{p} 2 \in[\mathrm{p}]$, if $\mathrm{p} 1$ has high similarity with $\mathrm{p} 2$ based on the similarity relationship $\mathrm{S}$ in $\mathrm{Wp}$, then $\mathrm{p} 1$ and $\mathrm{p} 2$ have high similarity in solution Ws. This idea indicates that decision makers can obtain the similar solution of the target case based on the decision of the similar case. In this paper, MOGA is applied to accomplish the process of case adaptation based on the similarity relation, in which object function is formed from case similarity function (CSF) and gray relational degree function (GRDF). The function of CSF is used for computing the similar degree, ensuring the case generated by MOGA has high similarity to current case. At the same time, GRDF aims to calculate the gray relational coefficients of independent variables and dependent variables. Then, it can be found that the design of CSF and MOGA meet the requirements of similarity theory for extending case base.

3.1.3 The Theory of BPNN for Decision-making

BPNN has been seen as a very powerful method for modeling complex non-linear systems benefited from its special biological structure. The reason why BPNN have strong ability in making decisions is that BPNN could determine the complex functional relations between input features and output results through continuous learning,

ISSN: 0010-8189

(C) CONVERTER 2021

www.converter-magazine.info 
which indicates the inner relations of dependent variables and independent variables.Initially, connections between different neurons are randomly given different weights to calculate decision feature value with the input scenario feature values. If the calculated decision feature value were different from the decision feature value in the sample, the weights would be adjusted according to designed model until the error difference between calculated values with that in the sample. Then, the weights of connections would be stored in the computer, indicating the relations between scenario features and decision feature have been determined.

\subsection{Methodology}

We followed the method of Zhang Baishang et al (2015) for the emergency management of wind damage to power grid [35]. Although the method of Zhang Baishang et al (2015) is ability to make decisions for wind damage by using Genetic Algorithm and Gray Relational Analysis, this method cannot make emergency plan quickly. Unlike the method proposed by Zhang Baishang et al (2015), this method focuses on making fast decisions by integrating CBR with RBR together.

\subsubsection{The Method of Extending Case Base}

3.2.1.1 The Method for Case Representation

It is known that there are many case representation methods, which includes method based on ontology, method based on tree structure and so on. In comparison with other methods, frame representation method has advantages in meeting the quantitative requirement because of its good structure and clear logic, which is proper for the case representation of power grid wind disaster emergency[35] However, a lot of incomplete information exists in power grid emergency, causing decision makers to estimate the accuracy of obtained information. D/S evidence theory are suitable for dealing with uncertain information because this theory is not requirement for obtaining prior probability and conditional probability, which is applied in target recognition, collective assessment and so on[36]. $\mathrm{D} / \mathrm{S}$ evidence theory assumes that $\mathrm{U}$ is a discernment frame of a complete set for incompatible events, being expressed as $\mathrm{U}=\left\{\theta_{1}, \theta_{2}, \ldots, \theta_{j} \ldots, \theta_{N}\right\}$, in which $\theta_{j}$ is an element of the discernment frame, $\mathrm{j}=1,2, \ldots, \mathrm{N}$; a set form from all of the subsets of the discernment frame is called the power of $\mathrm{U}$, which can be expressed as $2^{U}$. Then, two definitions can be obtained:

The first definition: Belief function Assume that there is a data set, including two data sets A and B, $\mathrm{B} \subseteq \mathrm{A} \subseteq \mathrm{U}$. If $m(B)$ is the basic probability assignment function of $\mathrm{B}$ and $\operatorname{Bel}(A)=\sum_{B \subseteq A} m(B)$, then Bel (A) is referred as the belief function of $\mathrm{A}$, which is a mapping from a set to $[0,1]$.

The second definition: Likelihood function Assume that $\mathrm{A}$ is a subset of $2^{U}$ and $\mathrm{P} *(\mathrm{~A})$ is a mapping from set $2^{U}$ to $[0,1]$. If $\mathrm{P}^{*}(\mathrm{~A})$ satisfies $\mathrm{P}^{*}(\mathrm{~A})=1-\operatorname{Bel}(\neg \mathrm{A})$, then $\mathrm{P}^{*}(\mathrm{~A})$ is called likelihood function.

According to above definitions the combination rules of incomplete information can be obtained as (3):

$$
m(A)=\left\{\begin{array}{cc}
\sum_{A_{i} \cap B_{i}=A} m_{1}\left(A_{i}\right) m_{2}\left(B_{j}\right)+\Delta A & A \neq \varnothing \\
0 & A=\varnothing
\end{array}\right.
$$

in which

$$
\Delta A=\sum_{A \cap B_{j}=\varnothing} \rho\left(A, B_{j}\right)+\sum_{A \cap A_{i}=\varnothing} \sigma\left(A, A_{i}\right)
$$

ISSN: 0010-8189 


$$
\begin{gathered}
\boldsymbol{\rho}\left(\boldsymbol{A}, \boldsymbol{B}_{j}\right)=\left\{\begin{array}{cc}
\frac{m_{1}(A) m_{1}(A) m_{2}\left(B_{j}\right)}{m_{1}(A)+m_{2}\left(B_{j}\right)} & m_{1}(A)+m_{2}\left(B_{j}\right)>0 \\
0 & m_{1}(A)+m_{2}\left(B_{j}\right)=0
\end{array}\right. \\
\sigma\left(A, A_{i}\right)=\left\{\begin{array}{cc}
\frac{m_{2}(A) m_{2}(A) m_{1}\left(A_{i}\right)}{m_{2}(A)+m_{1}\left(A_{i}\right)} & m_{2}(A)+m_{1}\left(A_{i}\right)>0 \\
0 & m_{2}(A)+m_{1}\left(A_{i}\right)=0
\end{array}\right.
\end{gathered}
$$

Therefore, the integration of frame case representation method and D/S evidence theory to represent emergency case in power grid wind disaster, which is constructed with nested frames. The nested frame includes a dependent variables sub-frame, an independent variables sub-frame and an effectiveness evaluation sub-frame and each frame has many slots. What important is that each slot can be divided into many sides with the basic probability assignment function (BPA) $\mathrm{m}$ (A) for describing reliability. The example of transmission line repairs in Eastern Shandong on August 3rd, 2012 is applied to describe how to represent the emergency case (See Table 1 to Table 4).

Table 1 The wind damage emergency main frame

\begin{tabular}{|l|}
\hline \multicolumn{1}{|c|}{ Main Frame Name:< wind damage emergency management for power grid > } \\
\hline Slot1: <disaster scenarios> \\
\hline Slot2: < disaster solutions> \\
\hline Slot3: < solutions evaluation> \\
\hline
\end{tabular}

Table 2 The sub-frame1 of accident features

\begin{tabular}{|l|l|l|l|l|}
\hline Frame Name: < accident features > & \multicolumn{3}{|l|}{} & \\
\hline Slot1: Breaking Time & Value & \multicolumn{2}{|l|}{$\begin{array}{l}\text { At 3:00 A.M on } \\
\text { August.3 2012 }\end{array}$} & m(A) \\
& & & \\
\hline Slot 2: affected area & Value & estern Shandong & m(A) & 1 \\
\hline Slot 3: specific characteristics & Value & plain & 1 \\
\hline Subslot31: landform & Value & 100 mm & m(A) & 1 \\
\hline Subslot32: rainfall & Value & 10 category & m(A) & 1 \\
\hline Subslot33: wind power & Value & 577 places & m(A) & 1 \\
\hline Subslot34: broken 10 KV lines & Value & 473 bases & m(A) & 1 \\
\hline Subslot35: impaired 10KV towers & Value & 0 place & m(A) & 1 \\
\hline Subslot36: broken 35KV lines & Value & 0 base & m(A) & 1 \\
\hline Subslot37: impaired 35KV towers & Value & 0 places & m(A) & 1 \\
\hline Subslot38: broken 66 KV lines & Value & 0 bases & m(A) & 1 \\
\hline Subslot39: impaired 66 KV towers & Value & 0 places & m(A) & 1 \\
\hline Subslot310: broken 110KV lines & & & \\
\hline
\end{tabular}

ISSN: 0010-8189 


\begin{tabular}{|l|l|ll|l|c|}
\hline Subslot311: impaired 110KV towers & Value & 0 bases & $\mathrm{m}(\mathrm{A})$ & 1 \\
\hline Subslot312: broken 220 KV lines & Value & 0 & places & $\mathrm{m}(\mathrm{A})$ & 1 \\
\hline Subslot313: impaired 220 KV towers & Value & 0 & bases & $\mathrm{m}(\mathrm{A})$ & 1 \\
\hline Subslot314: broken 330KV lines & Value & 0 & places & $\mathrm{m}(\mathrm{A})$ & 1 \\
\hline Subslot315: impaired 330KV towers & Value & 0 bases & $\mathrm{m}(\mathrm{A})$ & 1 \\
\hline Subslot316: broken 500 KV lines & Value & 0 places & $\mathrm{m}(\mathrm{A})$ & 1 \\
\hline Subslot317: impaired 500 KV towers & Value & 0 bases & $\mathrm{m}(\mathrm{A})$ & 1 \\
\hline Subslot318: broken 750 KV lines & Value & 0 places & $\mathrm{m}(\mathrm{A})$ & 1 \\
\hline Subslot319: impaired 750 KV towers & Value & 0 bases & $\mathrm{m}(\mathrm{A})$ & 1 \\
\hline
\end{tabular}

Table 3 The sub-frame of disaster solutions

\begin{tabular}{|c|c|}
\hline \multicolumn{2}{|l|}{ Frame Name: < Disaster solutions $>$} \\
\hline Slot1: crew & \\
\hline Subslot11: serviceman & 43368 \\
\hline Subslot12: support staff & 2398 \\
\hline Subslot13: motor vehicle and transit drivers & 5466 \\
\hline \multicolumn{2}{|l|}{ Slot 2: equipment } \\
\hline Subslot21: protective suits & 13104 \\
\hline Subslot22: mountaineering shoes & 0 \\
\hline Subslot23: gloves & 13104 \\
\hline Subslot24: rucksacks & 13104 \\
\hline Subslot25: mountaineering sticks & 0 \\
\hline Subslot26: headpiece & 13104 \\
\hline Subslot27: shoulder belts & 13104 \\
\hline Subslot28: intercoms & 426 \\
\hline Subslot29: binocles & 1274 \\
\hline Subslot210: line throwing guns & 0 \\
\hline Subslot211: climbing ropes & 0 \\
\hline Subslot212: bottles & 13104 \\
\hline Subslot213: emergency light & 10916 \\
\hline Subslot214: emergency searchlight & 1443 \\
\hline \multicolumn{2}{|l|}{ Slot 3: commodity } \\
\hline Subslot31: hard tack & 8890 \\
\hline Subslot32: fast food & 124.9 \\
\hline Subslot33: tabernacles & 4611 \\
\hline Subslot34: dinnerware & 451 \\
\hline
\end{tabular}

ISSN: 0010-8189 


\begin{tabular}{|l|l|}
\hline Subslot35: potable water & 165 \\
\hline Slot 4: other devices & \\
\hline Subslot41: electric generator & 4925 \\
\hline Subslot42: eletric torches & 1505 \\
\hline Subslot43: rescue vehicles & 12972 \\
\hline Subslot44: water pump & 8 \\
\hline Subslot45: motorboats & 58 \\
\hline Subslot46: portable tool boxes & 17454 \\
\hline
\end{tabular}

Table 4 The sub-frame of solutions evaluation

\begin{tabular}{|c|c|}
\hline \multicolumn{2}{|l|}{ Frame Name:< Solutions evaluation> } \\
\hline Slot1: The result & $\begin{array}{l}\text { By the seven o'clock the electric power supplying in } \\
\text { the affected area is restored, indicating the task of } \\
\text { fighting against typhoon to restore electric power is } \\
\text { fulfilled. }\end{array}$ \\
\hline Slot2: The deficiency of emergency plan & - \\
\hline Slot 3: The evaluation on transmission line repairs & $\begin{array}{l}\text { The emergency responding is high efficiency because } \\
\text { of the good cooperation of the government and } \\
\text { electric power companies and the proper operations. }\end{array}$ \\
\hline
\end{tabular}

\subsubsection{Retrieval Method}

The k-nearest neighbor method is used for retrieving similar cases after computing local similarity and global similarity.

(1) Local Similarity Computing

Different computing formulas are used to calculate local similarity because there are qualitative variables and quantitative variables in the decision process. For qualitative scenario features, if the scenario feature is different

from that of the target case, then the value of the similarity is 0 . Otherwise, the math type $e^{1-\left|m(A)_{01}-m(A)_{i 1}\right|}$ could be used for computing the local similarity. For quantitative scenario features, the local similarity could be calculated by (7):

$$
\operatorname{sim}_{j}=\left|1-\frac{\left|f_{0 j}-f_{i j}\right|}{\max \left(f_{0 j}, f_{i j}\right)}\right| *\left|m(A)_{0 j}-m(A)_{i j}\right|
$$

In above formulas, $m(A)_{0 j}$ and $m(A)_{i j}$ are BPAs of the scenario features in object cases and those in generated cases; $f_{0 j}$ and $f_{i j}$ are the attribute values of object case and those of generated cases.

(2) Global Similarity Computing

In comparison with analytic hierarchy process, G1 method can deal with problems with more than nine indexes, which is proper for determining the attributes of scenario features in power grid emergency case (Shi et al, 2014). According to much experience and professional knowledge, experts determine the order of the 19scenario features in transmission line repairs: $x_{4}^{*}=x_{5}^{*}=x_{6}^{*}=x_{7}^{*}=x_{8}^{*}=x_{9}^{*}=x_{10}^{*}=x_{11}^{*}=x_{12}^{*}=x_{13}^{*}=x_{14}^{*}=x_{15}^{*}=x_{16}^{*}=x_{17}^{*}=x_{18}^{*}=x_{19}^{*}>x_{2}^{*}=x_{3}^{*}>x_{1}^{*}$.Then, the formula 
based on the assignment principle $\mathrm{We}$ can be obtained: $r_{2}=x_{4}^{*} / x_{5}^{*}=1.0$, similarly, $r_{3}=r_{4}=r_{5}=r_{6}=r_{7}=r_{8}=r_{9}=r_{10}=r_{11}=r_{12}=r_{13}=r_{14}=r_{15}=r_{16}=r_{18}=1.0, r_{17}=x_{19}^{*} / x_{2}^{*}=1.4, r_{19}=x_{3}^{*} / x_{1}^{*}=1.2$.Then we can find $r_{2} r_{3} \cdots r_{19}=1.68, r_{18} r_{19}=r_{19}=1.2$. Finally, we have formula

$$
x_{19}^{*}=\left(1+\sum_{k=2}^{m} \prod_{i=k}^{m} r_{i}\right)^{-1}=(1+29.28)^{-1}=0.0330 \text {; we }
$$

can obtain the remaining values from $x_{17}^{*}$ to ${ }^{x_{1}^{*}}$ with the similar method. So we can get all the attribute weights (see Table 5).

Table 5 The attribute weights

\begin{tabular}{|l|l|l|l|l|l|l|l|l|l|l|l|l|l|l|l|l|l|l|}
\hline$x_{1}$ & $x_{2}$ & $x_{3}$ & $x_{4}$ & $x_{5}$ & $x_{6}$ & $x_{7}$ & $x_{8}$ & $x_{9}$ & $x_{10}$ & $x_{11}$ & $x_{12}$ & $x_{13}$ & $x_{14}$ & $x_{15}$ & $x_{16}$ & $x_{17}$ & $x_{18}$ & $x_{19}$ \\
\hline 0 & 0. & 0. & 0. & 0. & 0. & 0. & 0. & 0. & 0. & 0. & 0. & 0. & 0. & 0. & 0. & 0. & 0. & 0. \\
. & 0 & 0 & 0 & 0 & 0 & 0 & 0 & 0 & 0 & 0 & 0 & 0 & 0 & 0 & 0 & 0 & 0 & 0 \\
0 & 3 & 3 & 5 & 5 & 5 & 5 & 5 & 5 & 5 & 5 & 5 & 5 & 5 & 5 & 5 & 5 & 5 & 5 \\
3 & 9 & 9 & 5 & 5 & 5 & 5 & 5 & 5 & 5 & 5 & 5 & 5 & 5 & 5 & 5 & 5 & 5 & 5 \\
3 & 6 & 6 & 4 & 4 & 4 & 4 & 4 & 4 & 4 & 4 & 4 & 4 & 4 & 4 & 4 & 4 & 4 & 4 \\
\hline
\end{tabular}

So the formula for global similarity computing is obtained as (8):

$$
f 1= \begin{cases}0.0330 *|-| m(A)_{01}-m(A)_{i 1} \mid+\sum_{j=2}^{19} w_{j} \operatorname{sim}_{j} & j_{01}=j_{i 1} \\ \sum_{j=2}^{19} w_{j} \operatorname{sim}_{j} & j_{01} \neq j_{i 1}\end{cases}
$$

in which, $x_{1} \in[1,3], x_{2} \in[0,1055], x_{3} \in[0,16], x_{4} \in[0,300], x_{5} \in[0,300], x_{6} \cdots x_{19} \in[0,150]$, and the variables are integers above zero.

3.2.1.3 The Process of Multi-object Genetic Algorithm

According to above analysis, the multi-object functions include CSF and GRDF, which ensure the high similartiy relations at two aspects.

(1) CSF

CSF is used for ensuring the independent variables of the produced case has similar features as the target case, which is written as (9):

$$
f 1= \begin{cases}0.0330 * 1-\left|m(A)_{01}-m(A)_{i 1}\right|+\sum_{j=2}^{19} w_{j} \operatorname{sim}_{j} & j_{01}=j_{i 1} \\ \sum_{j=2}^{19} w_{j} \operatorname{sim}_{j} & j_{01} \neq j_{i 1}\end{cases}
$$

in which, $x_{1} \in[1,3], x_{2} \in[0,1055], x_{3} \in[0,16], x_{4} \in[0,300], x_{5} \in[0,300], x_{6} \cdots x_{19} \in[0,150]$, and the variables are integers above zero.

(2) GRDF

GRDF is ensuring dependent variables are obtained based on the corresponding independent variables(see Fig.2). If the obtained grey relational coefficient in the generated case has high similarity, then the outcomes in generated

ISSN: 0010-8189 
case have high accuracy.

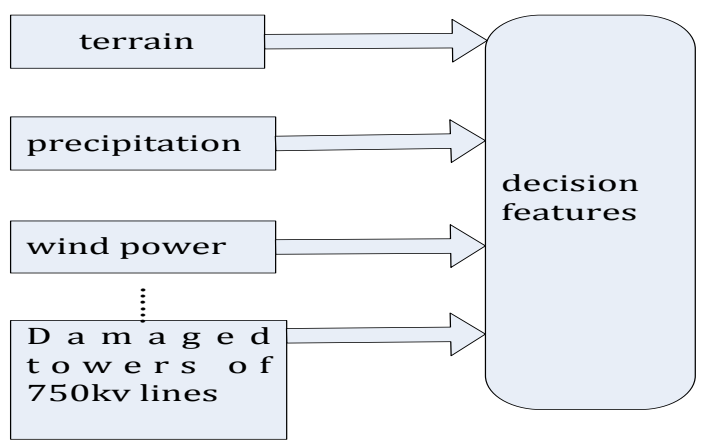

Fig 2: Functions between dependent variables and independent variables

In consideration of doing gray relational analysis (GRA) on GRDF, the theory of GRA is used to accomplish the process of GRDF modeling. The theory of GRA aims to build the corresponding relationship between dependent features and independent features in complex discrete systems on the condition of incomplete information and less data samples, which is proposed by Deng in 1982. In comparison with other methods, GRA is characterized by describing the correlation degree between objects with fewer samples. The GRDF model is built as the eight steps:

1) Building a 3 x 20 matrix with 19 independent variables and one independent variable.

2) Finding the main sequence $X_{0}$ and subsequences ${ }^{X_{i}}$.

3) Calculating absolute value of the difference between $X_{0}$ and $X_{i}$ at different times $\Delta_{i j}=\left|X_{0 j}-X_{i j}\right|$.

4) Computing the max value $\Delta_{\max }$ and min value $\Delta_{\min }$.

5) Calculating the connected relational coefficients between the main sequence and subsequences $r_{i j}=\frac{\Delta_{\min }+0.5 \Delta_{\max }}{\Delta_{i j}+0.5 \Delta_{\max }}$.

6) Working out all the correlation coefficients by using above method.

7) Adding the generated case to this matrix and building a $4 \times 20$ matrix. Calculating the grey correlation coefficients $S_{i j}$.

8) Calculate the sum of the differences between the two matrixes.

(3) The Multiple Object Functions

Multi-object functions include two targets:

$$
\left\{\begin{array}{l}
\max f 1, \\
\min f 2, \\
\text { s.t. } x_{1} \in[1,3], x_{2} \in[0,1055], x_{3} \in[0,16], x_{4} \in[0,300], \\
x_{5} \in[0,300], x_{6} \cdots x_{19} \in[0,150], x_{20} \cdots x_{49} \in[0,5000], \text { al I i nt eger } s \text { above zer o }
\end{array}\right.
$$

(4) Coding Method

This paper uses multi-parameter cascade to encode variables, which encodes the scenario features with binary code and encodes the decision features with real-number code.

(5) Initial Population

This paper uses five most similar cases for simulation.

(6) Selection

Roulette wheel selection method is used to select optimal polulation for each generation in this paper.

(7) Crossover

The adaptive GA has a critical problem: crossover is only finished on the chromosomes, causing a coarse partition

ISSN: 0010-8189

(C) CONVERTER 2021

www.converter-magazine.info 
[46]. Different from traditional methods, this paper improves the adaptive GA with (10):

$$
\mathbf{p}_{c}=\left\{\begin{array}{l}
\frac{p_{c 1}+p_{c 2}}{2}-\frac{\left(p_{c 1}-p_{c 2}\right)\left(f^{\prime}-f_{\text {avg }}\right)}{f_{\max }-f_{\text {avg }}}, \frac{f_{\max }+f_{\text {avg }}}{2} \leq f^{\prime} \leq f_{\max } \\
\frac{p_{c 1}+p_{c 2}}{2}-\frac{\left(p_{c 1}-p_{c 2}\right)\left(f^{\prime}-f_{\text {avg }}\right)}{f_{\max }-f_{\text {avg }}}, f_{\text {avg }}<f^{\prime}<\frac{f_{\max }+f_{\text {avg }}}{2} \\
\frac{p_{c 1}+p_{c 2}}{2}, f^{\prime} \leq f_{\text {avg }}
\end{array}\right.
$$

In above model, $\quad p_{c 1}=\frac{1}{2+\lg N}+\phi$ and $p_{c 2}=0.9$ are the respective maximum fitnesses for crossover.

(8) Mutation

The function of mutation in MOGA can be written as (12):

$$
\mathbf{p}_{m}=\left\{\begin{array}{l}
\frac{p_{m 1}+p_{m 2}}{2}-\frac{\left(p_{m 1}-p_{m 2}\right)\left(f^{\prime}-f_{\text {avg }}\right)}{f_{\max }-f_{\text {avg }}}, \frac{f_{\max }+f_{\text {avg }}}{2} \leq f^{\prime} \leq f_{\max } \\
\frac{p_{m 1}+p_{m 2}}{2}-\frac{\left(p_{m 1}-p_{m 2}\right)\left(f^{\prime}-f_{\text {avg }}\right)}{f_{\max }-f_{\text {avg }}}, f_{\text {avg }}<f^{\prime}<\frac{f_{\max }+f_{\text {avg }}}{2} \\
\frac{p_{m 1}+p_{m 2}}{2} \quad, f^{\prime} \leq f_{\text {avg }}
\end{array}\right.
$$

In this model, $\quad p_{m 1}=\frac{-0.1}{2+0.7 \lg N}+\varphi$ and $p_{m 2}=0.1$ are the maximum fitness.

(9) The Condition of Algorithm Stopping

This research will stop when 50 generations are finished.

3.2.2 BPNNASAA Training

3.2.2.1 BPNNASAA model

In general, the error function could be defined as (13)

$$
E=\frac{1}{2} \sum_{k=1}^{m}\left(y_{k}-o_{k}\right)^{2}
$$

In which $y_{k}$ and ${ }^{O}{ }_{k}$ represent the output values and the actual values in respective, and $\mathrm{k}$ is the output neuron. BPNN adopts the gradient descent algorithm to adjust the weights according to (14):

$$
\frac{\partial \boldsymbol{E}}{\partial w_{i j}}=\frac{\partial E}{\partial n e t_{j}} \cdot \frac{\partial n e t_{j}}{\partial w_{i j}}=\frac{\partial E}{\partial n e t_{j}} \cdot \frac{\partial\left(\sum_{k} w_{k j} o_{k}\right)}{\partial w_{i j}}=\frac{\partial E}{\partial n e t_{j}} \cdot o_{i}
$$

ISSN: 0010-8189 
In which $\frac{\partial E}{\partial n e t_{j}}$ could be written as $\delta_{j}$. At last, the equation for adjusted weights could be obtained as (15):

$$
\Delta w_{i j}=\alpha \delta_{j} o_{i}
$$

In which $\alpha$ is the learning rate, and $\delta_{j}$ denotes signal error what could be calculated according to the following conditions: If neuron $\mathrm{j}$ is one of the output neuron, then some equations from (16) to (19) could be obtained

$$
\begin{gathered}
\delta_{j}=\left(y_{j}-o_{j}\right) f^{\prime}\left(\text { net }_{j}\right) \\
w_{i j}^{j}=w_{i j}^{j-1}+\alpha f^{\prime}\left(\text { net }_{j}\right)\left(y_{j}-o_{j}\right) o_{i}
\end{gathered}
$$

Else

$$
\begin{gathered}
\delta_{j}=\left(\sum_{k=1}^{H_{k}} \delta_{k} w_{j k}\right) f^{\prime}\left(\text { net }_{j}\right) \\
w_{i j}^{j}=w_{i j}^{j-1}+\alpha f^{\prime}\left(\text { net }_{j}\right)\left(\sum_{k=1}^{H_{k}} \delta_{k} w_{j k}\right) o_{i}
\end{gathered}
$$

Although BPNN have advantages in optimum searching, it is confronted with slow convergence and local minimum what cause its application restriction. In order to improve the performance of BPNN, S. Szu proposes BPNNSAA based on Cauchy training what is built on the principle that energy variation would be calculated according to (20) after the condition that system is randomly given an initial state $\left\{w_{i j}^{(p)}\right\}$ and a small random disturbance $w_{i j}^{(p)}$ :

$$
\Delta E=E\left\{w_{i j}^{(p)}+\Delta w_{i j}^{(p)}\right\}-E\left\{w_{i j}^{(p)}\right\}
$$

If $\Delta E<0$, then the disturbance could be accepted; else, the disturbance would be accepted or refused according to $(21)$ :

$$
\exp \left(-\frac{\Delta E}{k T}\right)
$$

in which $\mathrm{k}$ is Boltzmann constant and $\mathrm{T}$ denotes artificial temperature. If the disturbance is accepted, then the system transforms from the state $\left\{w_{i j}^{(p)}\right\}$; else, the system state would not be changed. During the process, the temperature would get continuous decrease, indicating that the system are getting more close to the optimum. S. Szu's model replaces Boltzmann distribution by Cauchy distribution what could be written as (22):

$$
p(x)=-\frac{T}{T^{2}+x^{2}}
$$

The big advantage of Cauchy training is that more opportunities to get larger adjustment of connection weights would be increased, improving the performance in training speed.

Actually, the learning rate in S. Szu's model is usually set subjectively and tentatively, influencing optimization

ISSN: 0010-8189 
searching and convergence. Motivated by this defect, this paper proposed BPNNASAA to adjust learning rate automatically according to object function. The thought of BPNNASAA comes from the the metal annealing process, during which the process is divided into lots of stages according to energy variation. BPNN has stages in the training process similar to annealing process, during which stages artificial special heats could be defined according to the definition of special heat in the thermodynamics. In the boundary point of the energy variation, the artificial special heat, being defined as the average ratio of artificial temperature to object function varies greatly. During the process of starting period (the high temperature period) and the latter period (the low temperature period) in the training, energy varies in stably and the artificial special heat is almost a constant, indicating that the system is likely to be far from the local minimum. Then, the temperature could vary fast in the two stages to improve training speed. Conversely, when the system is in a critical temperature, the small decrease of temperature could cause large variation of the energy, meaning that the system is close to the local minimum. Then, the temperature should be decreased slowly in the critical temperature. Then, the mathematical formula of connection weights in the BPNNASAA could be written as (23):

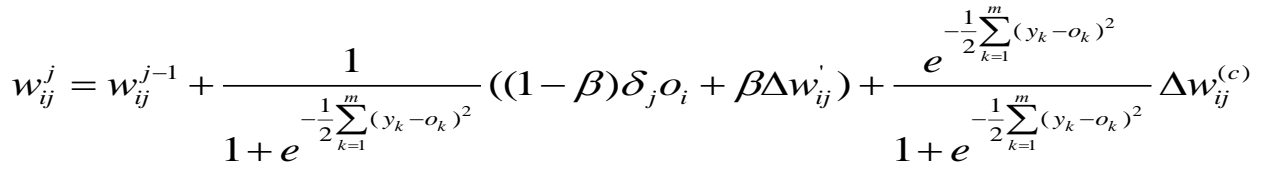

\subsubsection{BPNNASAA Building}

(1) Parameter Determination

ANN could be divided into homogenous ANN model and heterogeneous ANN according to difference in the input neurons. In the training process of heterogeneous ANN, multi-variable are input the model what considers all kinds of factors influencing the output value. Therefore, this paper uses 19 scenario features in the disaster emergency of power grid wind as the input neurons.

The numbers of layers in the ANN are determined by the numbers of hidden layers on the condition that the input layer and the output layer are both one layer. Theoretically, if there are enough neurons, the model could be ability to approximate to arbitrary continuous function. In empirical research, the ANN models with one hidden layer or two hidden layers have been applied extensively because of their excellent performance. Based on above analysis, this paper designed the one hidden layer BPNNASAA model.

The output neuron numbers need to be determined by the designer, which affects the performance of ANN model. Therefore, this paper selected each decision feature as the single neuron in the output layer of BPNNASAA, finally building 30 BPNNASAA with similar structures.

Some scholars proposes some empirical criterion for neuron number determination according to empirical comparative. Masters points out that the neuron number varies between $1.5 \sqrt{n \times m}$ and $2 \sqrt{n \times m}$ according to the actual conditions. Then, the neuron number in this research should vary from six to twelve theoretically. In order to determine the number more precisely, this paper maintained nine neurons in the hidden layer with empirical comparative method.

(2) Normalization of cases

The independent variables and dependent variables have been normalization with (24):

$$
N\left(\mathrm{x}_{i}\right)=\frac{x_{i}-x_{\min }}{x_{\max }-x_{\min }}
$$

In which $x_{\max }$ and $x_{\min }$ are the max value and the minimum value in the same feature respectively.

ISSN: 0010-8189 


\section{System simulation and Results Analysis}

Based on above theory and technologies a decision supporting system for power grid emergency is developed with Matlab, VC++ 6.0 and Microsoft Access to test the feasibility and validity of the developed system.

\subsection{System simulation}

The case of typhoon "Rainbow" hitting the power grid in Foshan on the 4th October, 2015 to test the validity of the developed system. When the typhoon arrived in Foshan, the magnitude of the wind in some regions were eight and the rainfall is $75 \mathrm{~mm}$, causing the power grid in Foshan to get severe damage. Two field exploration teams found that there were 153 damaged places on the $10 \mathrm{kv}$ lines by the typhoon, but the tower damage on the $10 \mathrm{kv}$ lines could not be exploited specifically. According to the time and space model of failure probability in the typhoon and storm for power grid, the teams reported the scenario information of the tower damage on the $10 \mathrm{kv}$ lines are $(60,0.95)$ and $(54,0.5)$ respectively. The basic probability assignment of other scenario features is 1 . According to D/S evidence theory, this paper deal with the uncertain information with equations from (25) to (27):

$$
\begin{gathered}
m(a)=0.95 \times 0+\frac{0.95 \times 0.95 \times 0.5}{0.95+0.5}+\frac{0.95 \times 0.95 \times 0.5}{0.95+0.5}=0.6225 \\
m(b)=0.5 \times 0+\frac{0.5 \times 0.5 \times 0.95}{0.95+0.5}+\frac{0.5 \times 0.5 \times 0.05}{0.5+0.05}=0.1865 \\
m(c)=0.05 \times 0.5+\frac{0.05 \times 0.05 \times 0.5}{0.05+0.5}+\frac{0.5 \times 0.5 \times 0.95}{0.5+0.95}=0.1910
\end{gathered}
$$

Then we can get the BPA of the tower damage on the $10 \mathrm{kv}$ lines whose value is 0.6225 . The scenario features of the example is represented as Fig.3.

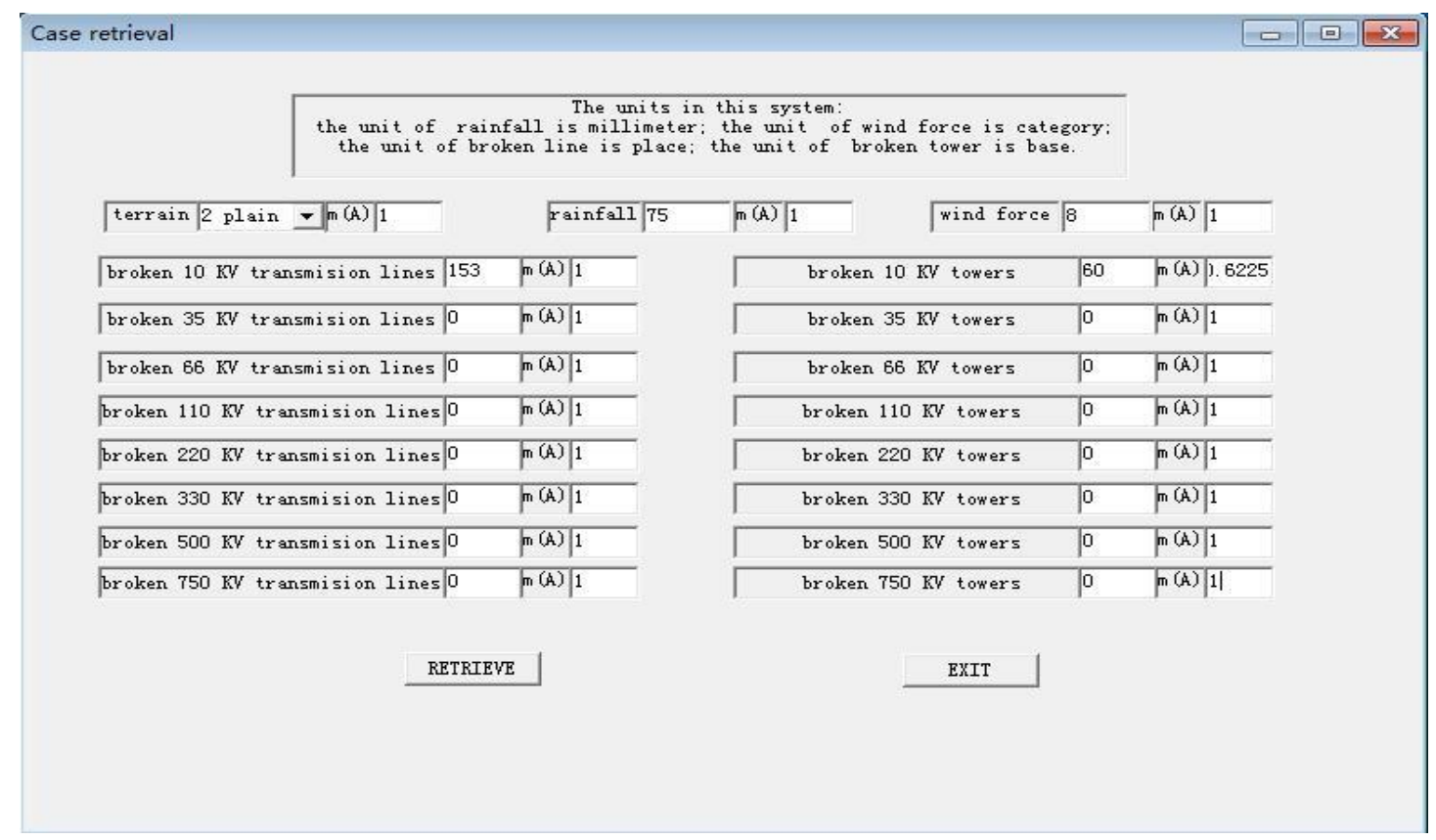

Fig 3: The case representation in the system

ISSN: 0010-8189 
This system retrieves similar cases with the nearest neighbor algorithm by implementing local similarity calculation and global similarity calculation. According to the calculation method of qualitative attributes, the local similarity degree of the symbol attribute "terrain" is computed by (28):

$$
1-|1-1|=1
$$

The similarity of quantitative attributes is calculated with (7). For example, the similarity degree of "rainfall" is computed as (29).

$$
|1-| \frac{100-75}{546-80}|| \times|1-| 1-1||=0.9464
$$

The values of the rest local similarities are $0.75,0.2083,0.6711,1,0.8966,1,1,1,1,1,1,1,1,1,1,1,1$. Then, the global similarity of the most similar case can be calculated with G1 method, whose value is 0.9188 .

It is found that the quantity of the retrieved similar cases are less for case adaptation, activating the component of case base expansion to produce more similar cases. After producing plenty of cases, the system complements the case adaptation process with parallel structure of BPNNASAA, outputting the emergency plan as Fig.5.

\subsection{Effectiveness Evaluation}

\subsubsection{Research Analysis}

\subsubsection{Application Analysis}

The developed system has the function of outputting pictures for analyzing the accuracy of the results, displaying the adaptability of object function 1 (OF1) and object function (OF) (see Fig.4). It is seen that the value of OF1amountsto 1 at generation 300 and the value of OF2 is almost 127 when the algorithm stops (see Fig.5).

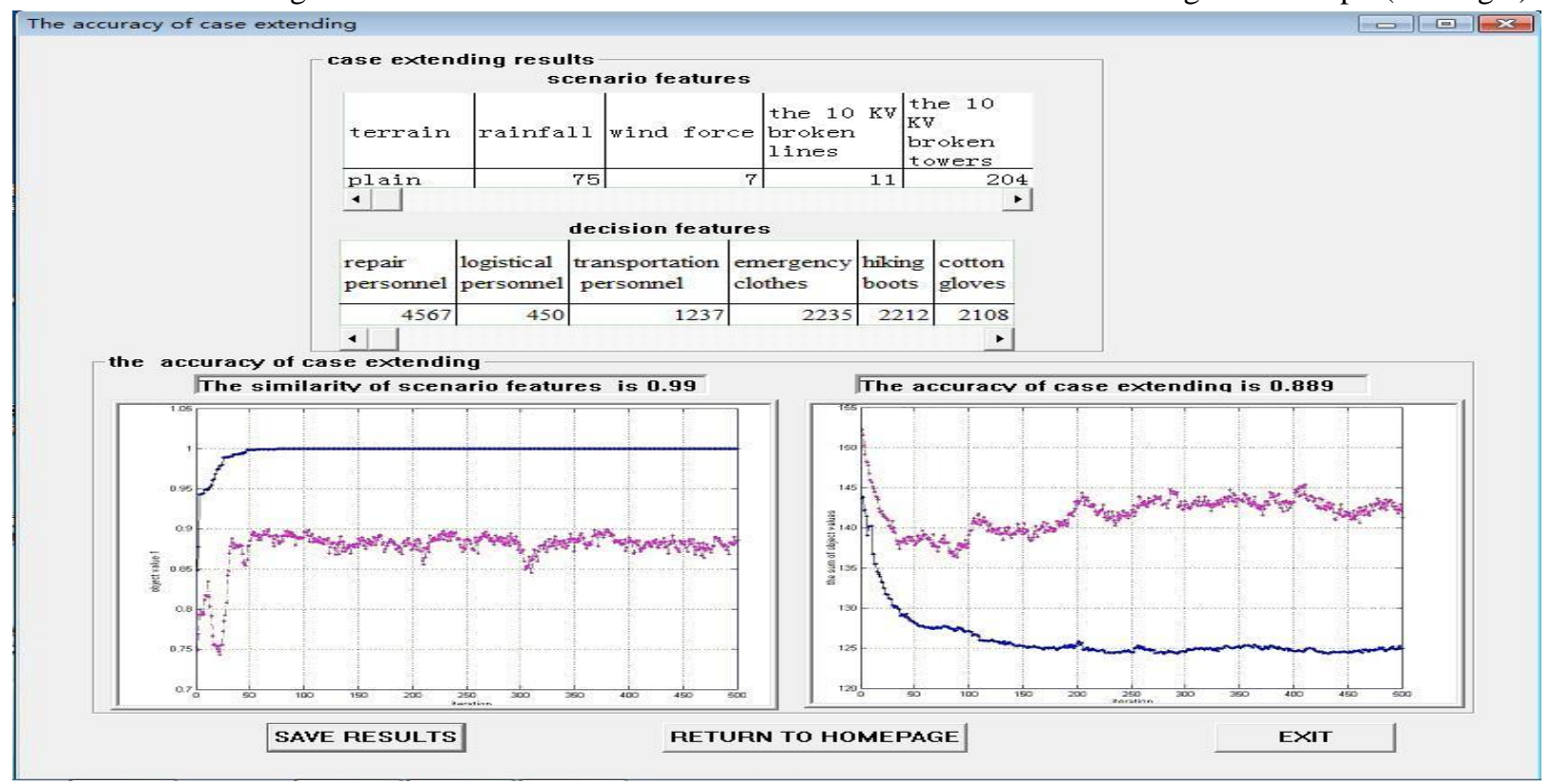

Fig 4: Application analysis for GRAMOGA

ISSN: 0010-8189 


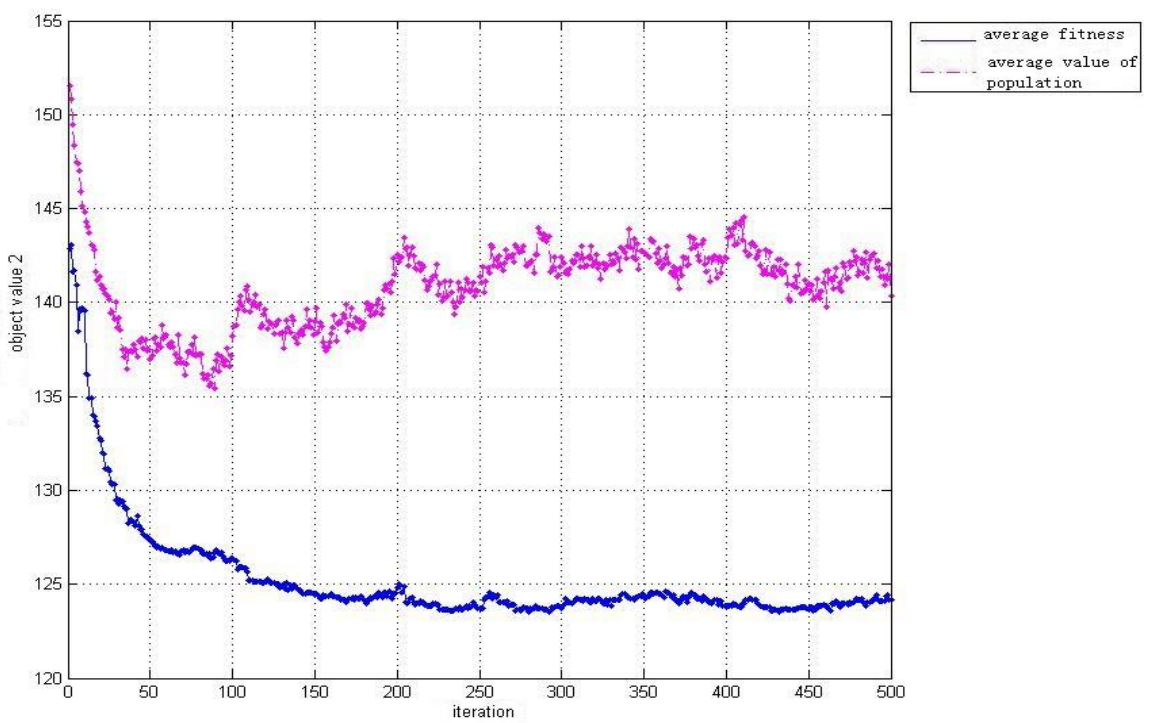

Fig 5: The convergence of OF2 in GRAMOGA

According to above consideration, if the results are not right at all, then the value of OF2 will be 570 . So the adaptability of OF2 is $(1-127 / 570)=0.778$, meeting the requirements of decision making accuracy. Based on the adaptability of OF1 and OF2, this paper gets the globle accuracy of adaptability whose value is $(1+0.778) / 2=0.889$. 4.2.1.2 The Analysis of Convergence

From Figure 4 to Figure 7 it can be seen that the OF1 in SGA could not converge and its searching result is 0.96 ; GRAMOGA gets the state of convergence with less generation steps, whose value of the optimal solution is 1 . Similarly, the OF2 in SGA cannot get the target of convergence either, whose optimal value is 137; the OF2 in GRAMOGA can converge in less time, whose searching value is 16 , showing better searching efficiency.

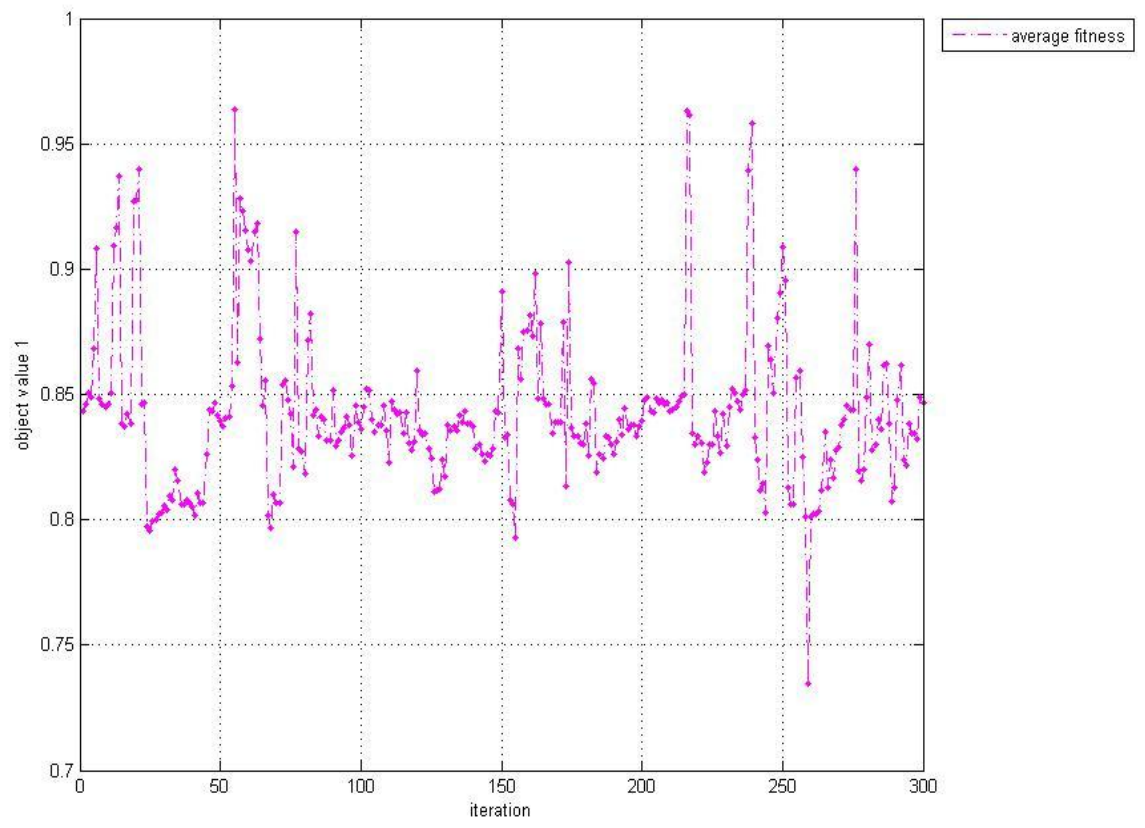

Fig 6: OF1Convergence of SGA

ISSN: 0010-8189 


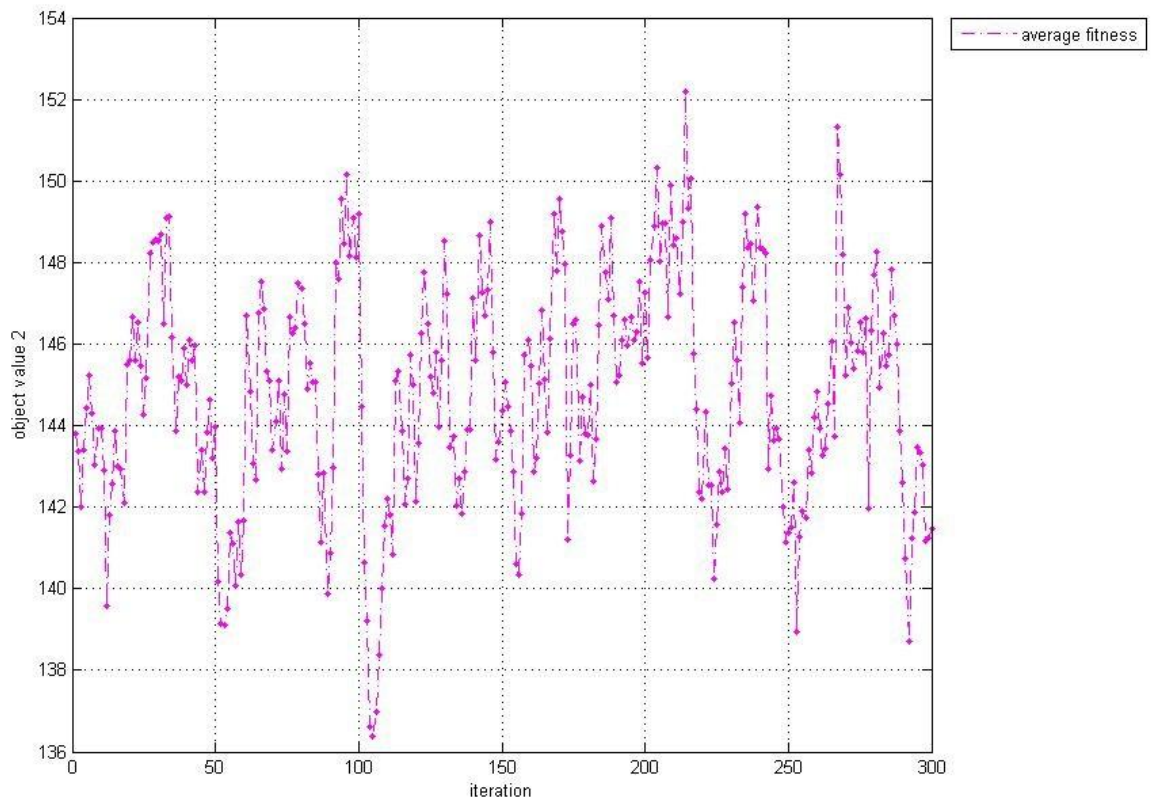

Fig 7: OF2Convergence of SGA

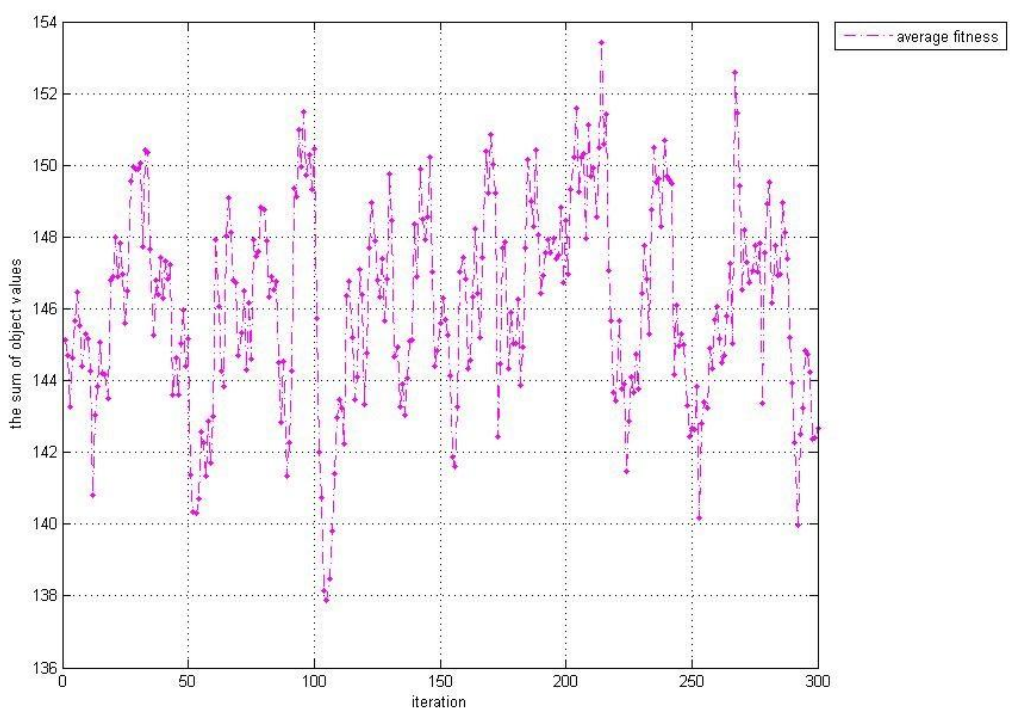

Fig 8: OF Convergence of SGA

Similarly, we can compare the performance of the OF in SGA and that in GRAMOGA. It can be seen that SGA could not get the state of convergence, whose searching value is 138; GRAMOGA can converge with high speed and obtain the optimal solution whose value is 127.

From above comparison, it is clearly found that the GRAMOGA is more better in performance.

\subsubsection{Analysis of BPNNASAA}

Usually the accuracy analysis of BPNN could be made with equations from (30) to (32):

ISSN: 0010-8189

(C) CONVERTER 2021 


$$
\begin{gathered}
\text { RMSE }=\sqrt{\frac{\sum(y-o)^{2}}{N}} \\
\text { Bias }=\frac{\sum(y-o)}{N} \\
R^{2}=\frac{N \sum_{i=1}^{N} o_{i} y_{p i}-\left(\sum_{i=1}^{N} o_{i}\right)\left(\sum_{i=1}^{N} y_{p i}\right)}{\left[N \sum_{i=1}^{N} o_{i}^{2}-\left(\sum_{i=1}^{N} o_{i}\right)^{2}\right] \times\left[N \sum_{i=1}^{N} p_{i}^{2}-\left(\sum_{i=1}^{N} p_{i}\right)^{2}\right]}
\end{gathered}
$$

After 5000 training iteration, the accuracy is e-8, indicating that the high performance has been achieved with BPNNASAA. The accuracy evaluation is illustrated in the Fig.9 what shows RMSE, Bias and R denote the network error, the dependent error and the relations between dependent variables and independent variables respectively. Clearly, BPNNASAA obtained high accuracy with low network error and variables error, meeting the requirements of fast decision making.

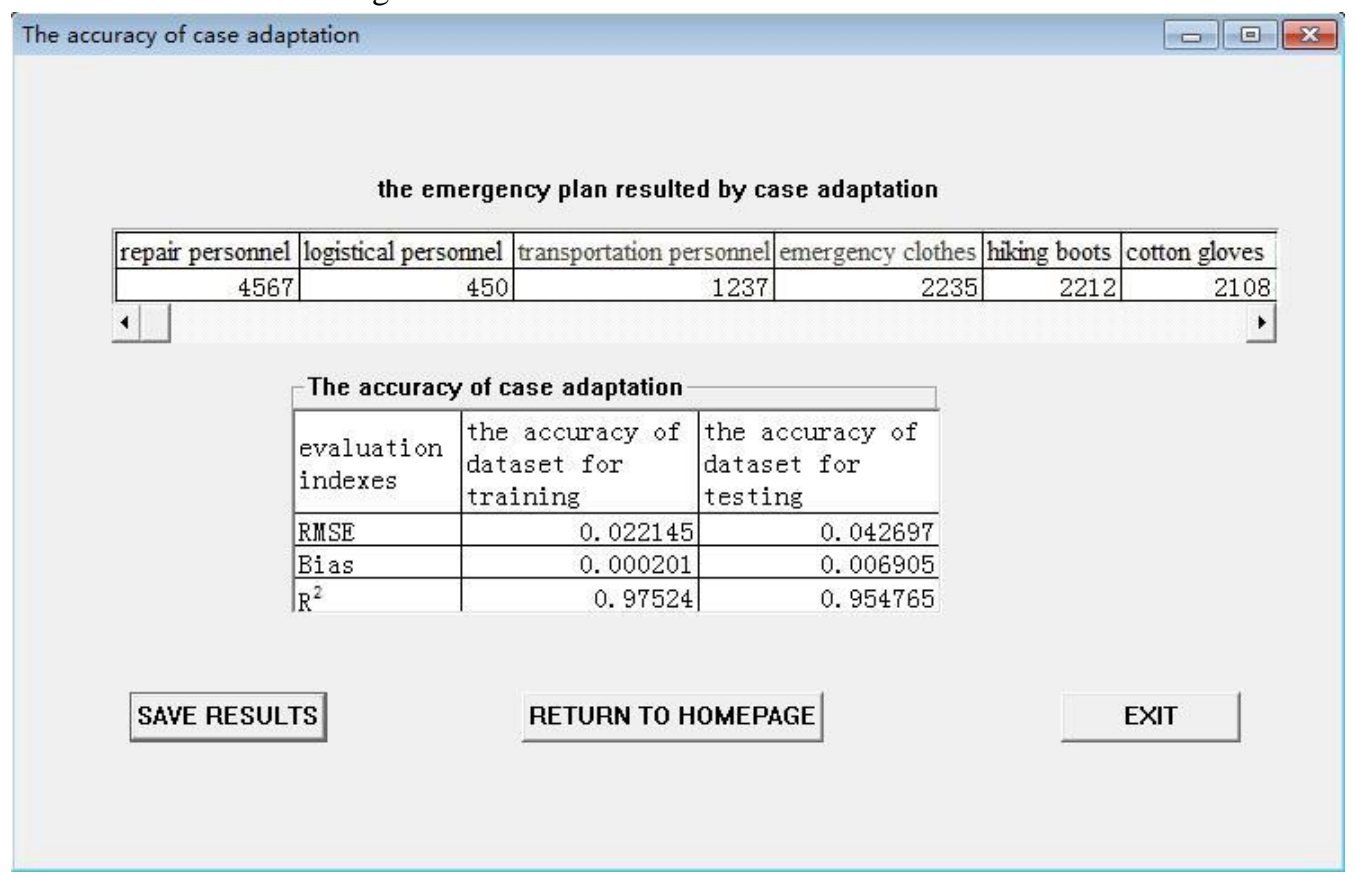

Fig 9: Application analysis for BPNNASAA

\section{Discussion}

There are many complex functions what could not be described with mathematical formula, causing traditional decision-making methods to be useless. Motivated by the advantages of integrated intelligent technologies, this paper integrates CBR, GRAMOGA and BPNNASAA together to let the intelligent methods help each other. Specifically, although CBR is suitable to deal with the complex non-line system problem, the case adaptation in CBR needs the help of BPNNASAA because the sole CBR is almost unable to accomplish case adaptation and BPNNASAA has the ability in case adaptation through constructing complex models.Clearly, CBR, GRAMOGA and BPNNASAA forms a whole unity what can work with high efficiency, but the unity will break down if any one module is lost.

ISSN: 0010-8189 
One characteristics of this system is that it have good ability in dealing with the incomplete information, which is very helpful for decision making under the condition of urgency and uncertainty. In this paper, two sources of incomplete information will be fused with $\mathrm{D} / \mathrm{S}$ evidence theory for accuracy decision-making, increasing the ability of this system in dealing with problems with incomplete information. However, the fusion of three and more sources of incomplete information has not been proposed in this paper, which is very common in the reality, decreasing the application ability of this system. Then, the further research is to explore the method of dealing with three and more sources of incomplete information for accuracy decision-making.

\section{Conclusion}

Based on FDMMIIT, a novel method for fast decision-making in wind damage emergency management for power grid is proposed. To focus on the combination effect of independent variables on independent variables, this paper proposes FDMMIIT with CBR, GRAMOGA and BPNNASAA. Importantly, a novel selection method are used for improving SGA and BPNNSA has been modified by using adaptive learning rate what could be adjusted according to the energy function. Furthermore, the paper integrates the frame representation method and D/S evidence theory for dealing with information fusion. At last, this method is tested with real case in China. Compared with previous research, the contributions of this article are ( $i)$ this paper designed a quick decision method for typhoon damage management by integrating CBR, GRAMOGA and BPNNASAA together to form a whole decision making unity, which has more advantages than traditional decision making methods in dealing with complex non-line system problem, fast decision making and incomplete information; (ii) Parallel structure of BPNNASAA is designed to finish case adaptation, which has high accuracy in case adaptation and enriches the case adaptation methods; (iii) A novel case base extending methods based on GRAMOGA is proposed to produce new cases for the case adaptation in this system. In comparison with case base extending methods based on RBR or Mente Carlo Method, GRAMOGA has ability in extending both qualitative case base and quantitative case base, whose prominent advantages includes operable steps and verifiable testing performance. (IV)This research has done some improvements in BPNNSAA and SGA FOR high accuracy. For the performance improvement of BPNNSAA, this paper proposed BPNNASAA to avoid the randomness and tentative in BPNNSAA by adjusting the energy function adaptively, whose high accuracy is veritified.

However, there are defects in the method. Firstly, this paper only proposes the methods of information fusion from two sources, exploring the information fusion with multi-source data. Secondly, fuzzy information what is very common in the reality is not considered in this paper, which causes the research to be limited in the application. Thirdly, the technology of decision-making with mobile device is not studied, which causes the research to move behind the current situation of intelligent technologies. In the future, this research will be extended in the information fusion with multi-source data, fuzzy decision-making and decision making with mobile devices.

\section{Acknowledgement}

Thanks for writing assistance and language help from Professor Tang and his students.

\section{Funding}

The paper is supported by the Major Project of National Social Sciences Fund (No.14ZDB166), the National Science Fund of China (No. 71774068 and 71303093), the Chinese Post-doctoral Science Fund (No.2016M590845) and the Fundamental Research Funds for the Central Universities of China (No.21612301).

\section{References}

[1] Qi. J, Hu. Jieg, Peng. Y. G, Incorporating Adaptability-related Knowledge into Support Vector Machine for Case-based Design Adaptation. Engineering Applications of Artificial Intelligence, 2015,

ISSN: 0010-8189

(C) CONVERTER 2021

www.converter-magazine.info 
37:170-180.

[2] Z. L. Liao, X. W. Mao, Phillip M. Hannam, Tingting Zhao. Adaptation methodology of CBR for environmental emergency preparedness system based on an Improved Genetic Algorithm. Expert Systems with Applications, 2012, 39(8):7029-7040.

[3] Qi. J, Hu. J, Peng. Y. G, A New Adaptation Method Based on Adaptability Under k-nearest Neighbors for Case Adaptation in Case-based design. Expert Systems with Applications, 2012, 39(7):6485-6502.

[4] Reader Arthur V. Unclear memory: Review of Dynamic Memory: A Theory of Reminding and Learning in Computers and People, by Schank Roger C. Cambridge, U.K.: Cambridge University Press, 1982. Pergamon, 1988, 6(1).

[5] Ping Xiaoou, Tseng Yiju, Lin Yanpo, etal. A Multiple Measurements Case-Based Reasoning Method for Predicting Recurrent Status of Liver Cancer Patients. Computers in Industry, 2015, 69 12-21.

[6] Teodorović Dušan,Šelmić Milica ,Mijatović-Teodorović Ljiljana .Combining Case-Based Reasoning with Bee Colony Optimization for Dose Planning in Well Differentiated Thyroid Cancer Treatment. Expert Systems with Applications, 2013, 40(6):2147-2155.

[7] Hong Taehoon, Koo Choongwan, Park Sungki. A Decision Support Model for Improving A Multi-family Housing Complex Based on CO 2 Emission from Gas Energy Consumption. Building and Environment, 2012, 52:142-151.

[8] Yeow Wei Liang, Mahmud Rohana, Raj Ram Gopal. An Application of Case-Based Reasoning with Machine Learning for Forensic Autopsy. Expert Systems with Applications, 2014, 41(7) 3497-3505.

[9] Esmat Rashedi, Nezamabadi-pour Hossein, Saryazdi Saeid. Long Term Learning in Image Retrieval Systems Using Case Based Reasoning. Engineering Applications of Artificial Intelligence, 2014, 35:26-37.

[10] Amailef Khaled, Lu Jie. Ontology-Supported Case-Based Reasoning Approach for Intelligent M-Government Emergency Response Services. Decision Support Systems, 2013, 55(1).79-97.

[11] Zhu Guoniu, Hu Jie, Qi Jin, etal. An Integrated Feature Selection and Cluster Analysis Techniques for Case-Based Reasoning. Engineering Applications of Artificial Intelligence, 2015, 39:14-22.

[12] Li Hui, Sun Jie. Hybridizing Principles of the Electre Method with Case-Based Reasoning for Data Mining: Electre-CBR-I and Electre-CBR-II. European Journal of Operational Research, 2008, 197(1):214-224.

[13] Pinzón Cristian I, De Paz Juan F, Herrero Álvaro, etal. idMAS-SQL: Intrusion Detection Based on MAS to Detect and Block SQL Injection Through Data Mining. Information Sciences, 2013, 231:15-31.

[14] Chuang Chunling. Case-Based Reasoning Support for Liver Disease Diagnosis. Artificial Intelligence in Medicine, 2011, 53(1).15-23.

[15] Lam H.Y., Choy K.L., HoG. T. S. AReal-Time Risk Control and Monitoring System for Incident Handling in Wine Storage[J].Expert Systems with Applications,2013, 40:3665-3678.

[16] Platon Radu, Dehkordi Vahid Raissi, Martel Jacques. Hourly Prediction of a Building's Electricity Consumption Using Case-Based Reasoning, Artificial Neural Networks and Principal Component Analysis. Energy \& Buildings, 2015, 92:10-18.

[17] Koo Choongwan, Hong Taehoon. A Dynamic Energy Performance Curve for Evaluating the Historical Trends in the Energy Performance of Existing Buildings Using a Simplified Case-Based Reasoning Approach. Energy \& Buildings, 2015, 92: 338-350.http://doi.org/10.1016/j. enbuild. 2015. 02.004.

[18] Lv Zheng, Liu Ying, Zhao Jun, etal Soft Computing for Overflow Particle Size in Grinding Process Based on Hybrid Case Based Reasoning. Applied Soft Computing Journal, 2015, 27:533-542.

[19] Yan Aijun, Shao Hongshan, Guo Zhen. Weight Optimization for Case-Based Reasoning Using Membrane Computing. Information Sciences, 2014, 287:109-120.

[20] Smiti Abir, Elouedi Zied. WCOID-DG: An Approach for Case Base Maintenance Based on Weighting, Clustering, Outliers. Journal of Computer and System Sciences, 2014, 80(1):27-38.

[21] Juan A. Recio-García, Miguel A. Casado-Hernández, Belén Díaz-Agudo. Extending CBR with Multiple Knowledge Sources from Web. Proceedings of 18th International Conference on Case-Based

ISSN: 0010-8189

(C) CONVERTER 2021

www.converter-magazine.info 
Reasoning, Springer Berlin Heidelberg, 2010, pp.287-301.

[22] Thomas Roth-Berghofer, Benjamin Adrian, Andreas Dengel. Case Acquisition from Text: Ontology-Based Information Extraction with SCOOBIE for My CBR. Proceedings of 18th International Conference on Case-Based Reasoning, Springer Berlin Heidelberg, 2010, pp.451-464.

[23] Arnolda Uwe, Yildiz Özgür. Economic Risk Analysis of Decentralized Renewable Energy Infrastructures-A Monte Carlo Simulation Approach. Renewable Energy, 2015, 77:227-239.

[24] Prentzas Jim, Hatzilygeroudis Ioannis. Incrementally Updating A Hybrid Rule Base Based on Empirical Data. Expert Systems, 2007, 24(4):212-231.

[25] Finnie. Gavin, Sun. Z. H, Similarity and Metrics in Case-Based Reasoning. International Journal of Intelligent Systems, 2002, 17(3):273-287.

[26] Sun. Z. H, Finnie Gavin, Weber Klaus. Case Base Building with Similarity Relations. Information Sciences, 2003, 165(1):21-43.

[27] Xu. M, Wong. T. C, Chin K. S. Modeling Daily Patient Arrivals at Emergency Department and Quantifying the Relative Importance of Contributing Variables Using Artificial Neural Network. Decision Support Systems, 2013, 54(3):1488 -1498.

[28] Lau H.C.W., Ho G.T.S, Zhao Yi. A Demand Forecast Model Using A Combination of Surrogate Data Analysis and Optimal Neural Network Approach. Decision Support Systems, 2013, 54(3).1404-1416.

[29] Yolcu Ufuk, Egrioglu Erol, Aladag Cagdas H. A New Linear \& Nonlinear Artificial Neural Network Model for Time Series Forecasting. Decision Support Systems, 2013, 54(3):1340 - 1347.

[30] Ma. G. Z, Song. E. M, Hung. C. C, etal. Multiple Costs Based Decision Making with Back-Propagation Neural Networks. Decision Support Systems, 2011, 52(3):657 -663.

[31] Chan Felix T.S, Chong Alain Y.L. A SEM-Neural Network Approach for Understanding Determinants of Interorganizational System Standard Adoption and Performances. Decision Support Systems, 2011, 52(3):621-630.

[32] Wang. S. L, Zuo. X. Q, Liu. X. Q, etal. Solving Dynamic Double Row Layout Problem via Combining Simulated Annealing and Mathematical Programming. Applied Soft Computing, 2015, 37:303 -310.

[33] Xie. X. L, Lin. L, Zhong. S. S, Handling Missing Values and Unmatched Features in A CBR system for Hydro-Generator Design. Computer-Aided Design, 2013, 45(6):963-976.

[34] Aliev. Rafik, Pedrycz. Witold, Fazlollahi. Bijan, etal. Fuzzy Logic-Based Generalized Decision Theory with Imperfect Information. Information Sciences, 2011, 189.18-42.

[35] Zhang Baishang, Li Xiangyang, Wang Shiying. A Novel Case Adaptation Method Based on An Improved Integrated Genetic Algorithm for Power Grid Wind Disaster Emergencies. Expert Systems with Applications, 2015, 42(21):7812-7824.

[36] Ghasemi. J, Ghaderi R, Karami Mollaei M.R, etal. A Novel Fuzzy Dempster-Shafer Inference System for Brain MRI Segmentation. Information Sciences, 2013, 223.205-220.

ISSN: 0010-8189 